\title{
Allocation of Greenhouse Gas Emissions for Containers in Multimodal Transshipment Terminals using Simulation
}

\author{
Uwe Clausen*, Moritz Poeting \\ Institute of Transport Logistics, TU Dortmund Unversity, Leonhard-Euler-Str. 2, 44227 Dortmund, Germany; \\ *clausen@itl.tu-dortmund.de
}

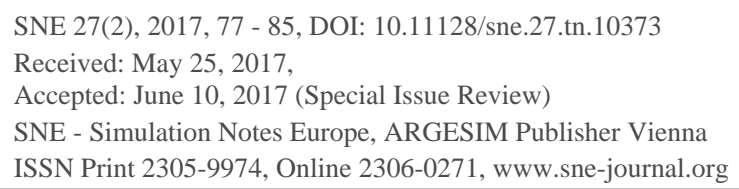

\begin{abstract}
The assessment of emissions caused by logistics operations in general and their allocation to individual customers in particular are major challenges for logistics service providers. Presently, numerous standards and guidelines exist (e.g. ISO 14064-1, ISO 14065, DIN EN 14040, DIN EN 14044, DIN EN 16258, PAS 2050) for the calculation of Greenhouse Gas (GHG) emissions caused by logistics processes. To support container terminal operators to determine their emissions, we use simulation to allocate overall emissions in a container terminal to a single container handling. To approach this goal, at first this paper describes the measurement of energy consumption from the handling equipment. After that, the paper shows the simulation approach to allocate the emissions.
\end{abstract}

\section{I ntroduction}

The climate change and its numerous negative influences to the development of weather are ascribed to the emission of greenhouse gases (GHG), especially carbon dioxide $\left(\mathrm{CO}_{2}\right)$. Those are identified as climatedamaging. To reduce these emissions and to counteract to the climate change national and international arrangements are made.

Since the global emission is growing continuously, Germany reduced its emissions in total to the target values which were assigned in the Kyoto protocol. Umweltbundesamt states that the transportation sector reduced its emissions since 1990 by $5.1 \%$.
The overall GHG-emissions of Germany's economy sectors were reduced by $21 \%$. In contrast to transportations, manufacturing reduced its emissions by $34.9 \%$ in the same time as well as private households by $33.1 \%$ and the energy sector by $14.8 \%$ [22]. Those numbers show the minor ratio of transportations concerning the reduction of $\mathrm{CO}_{2}$-emission. If only transportation is considered the emission is even increased by $13 \%$ regarding 1995 to 2010 [21].

Transportation is a substantial sector regarding the GHG-emissions and got a ratio of $20 \%$. Only the energy sector got a higher impact to the total GHGemission. So, realization of successful arrangements becomes essential. The increasing GHG-emission caused by transportation is not only obvious in Germany. It is also globally identifiable. Statistics, published by the Organization for Economic Cooperation and Development show that the total amount of GHGemission declines from 1990 to 2007 by $2 \%$ but on the other hand they increased by $45 \%$ in the transportations sector [17]. Also, the European Comission verifies this trend. Even though greenhouse gas emissions from transportation started to decrease in 2008, they still exeeded the target emission from the 2011 Transport white paper target by $67 \%$ in 2012 [9].

A reason for the difficulties to reduce GHGemissions concerning the transportation sector is the continuing growth of transport volume. Especially the freight traffic via road grew in recent times while simultaneously other modes of transport lowered their volume of ton kilometers.

This claims an analysis of Bundesverband Güterkraftverkehr Logistik und Entsorgung (BGL) e.V., illustrated in Figure 1. The analysis shows the distribution of ton kilometers concerning the modal split in freight transportation. 


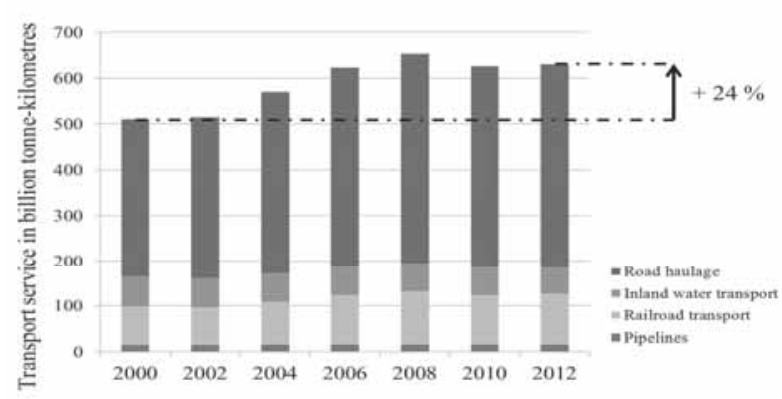

Figure 1: Modal Split.

Remarkable is that road transportation showed an allover volume share of $70.5 \%$ ton kilometers. This is a plus of $24 \%$ in comparison to the modal split share in 2000 [1]. This leads to higher increase of GHGemission caused by the transportation sector. A chance to counteract that trend is to shift road transportation to other modalities. This is called modal shift.

Modal shift aims at reducing the amount of road transportation and replace it with rail or barge transport. The main concept includes continuous transport using different modes of transportation in a single load unit from source to sink. But unnoted which kind of modality is chosen for transportation it will cause GHGemission.

It is necessary to allocate emissions caused by operations along the transportation chain. This is already possible for individual transportation modes. The emissions of related handling operations are usually estimated on the total consumption data of the whole facility. Information of handling emissions of loading units is insufficient. Regarding these circumstances, a detailed calculation of emission data is required to be able to allocate $\mathrm{CO}_{2}$-emissions for handling operations in multimodal container terminals.

In this article an approach is presented to evaluate GHG-emissions of container handling procedures in multimodal container terminals precisely.

\section{Related Works}

\subsection{Allocation of GHG-emissions}

Balancing GHG Emissions (which means $\mathrm{CO}_{2}, \mathrm{CH} 4$, N2O, HFC's, PFC's, SF6) is called CARBON FOOTPRINT and is part of the ecological balance sheet.
This ecological balance sheet is an instrument which allows calculating emissions of different products (goods and services) to make them comparable. The Norms [5,6] contain principles and examination framework for environmental accounting as well as methodological requirements for performing environmental accounting measures. The ecological balance sheet refers to environmental aspects and environmental effects which occur during product life cycle, originating from raw material about production, application, waste treatment to final disposal. According to the used method for impact assessment there can be considered up to fourteen impact categories. One of these categories is GHG-capability.

Detrimental in developing an ecological balance sheet is that even simple systems require a large data base which causes remarkable acquisition effort. Therefore, systems need to be simplified to minimize data needs. Though results do not reproduce an accurate image of the current situation, logistics carbon footprint can be determined for a product (good and service) as well for companies, a location, an organization or various transport services. Several methods, norms, guidelines and studies $[13,16,20]$ exist which set branchspecific basic conditions for assessing logistic processes and systems.

A norm for calculation and reporting of energy consumption and GHG-emissions of transport services (freight and passenger transport) has been initiated by the European Commitee for Standardisation (CEN) in 2008 [6]. The norm contains a standardized procedure for determining GHG-emissions for every of transport (road, rail, water and air traffic) along the entire supply chain (from shippers to forwarding agent and freight carrier up to subcontractors) and furthermore guidelines for standardized documentary. Additionally, it contains recommendations for determination of an adequate database. At least the user is free to choose individual measured values, vehicle/ route specific average or fleet values of transport service provider and defaults of database, although results differ in degree of detail.

However, those standards do not contain administrative or supportive operations (e.g. production planningand control process, maintenance, disposal) and stationary processes, like internal handling operations, so that essential elements are not included in balance results.

Basically, GHG-emissions of stationary logistic processes at multimodal logistic hubs are caused by following factors: 
- Power consumption of handling facilities, terminals, storage areas or offices

- Heat energy consumption

- Further used energy sources (e.g. gas, diesel fuel) for additional equipment like Reach Stackers, other terminal crafts or forklift trucks.

Since stationary processes were explicitly excluded in the currently existing standard EN 16258, in accordance with the Greenhouse Gas Protocol, the direct GHG emissions which occur through combustion of fuels as well as through occupation of electricity and heat are determined by using formula (1).

$$
\begin{gathered}
G H G=E C \cdot E F \\
E C: \text { Energy Consumption } \\
\text { EF:Emission Factor }
\end{gathered}
$$

Due to missing valid energy consumption data of multimodal handling terminals calculation is needed to be based on approved sources and estimations of energy consumption. Standards, guidelines, literature, manufacturer information and company details may be considered. However, sources do not provide consumption values for every single functional unit and existing information exclude terrain conditions or terminal layout within consumption data. This contribution points out in which way energy consumption values are allocated on loading unit level by using energetic simulation based on selective measurements energetic simulation in combined transport simulation is a preferred instrument for examination and evaluation of operations in course of time within multimodal handling terminals $[8,14,15]$.

\subsection{Simulation of energy consumption in multimodal transport}

Simulation solutions for holistic depiction of multimodal terminals have been developed by authors of previous articles. Those simulation solutions, based on material flow oriented simulation software Enterprise Dynamics, contain solutions for detailed planning of terminal systems as well as a low-level detailed solution for draft planning of multimodal handling terminals.

Those solutions contain modules for depiction of multimodal terminals and mathematical heuristics for optimal control of handling equipment, allocation of loading points and yard-management [3, 4, 10, 11, 12] or simulative descriptions of Carbon Footprints in supply chains or great logistic networks $[18,19]$.
As shown in those papers, current existing research projects and solution approaches in case of energetic simulation focus transport and exclude handling facilities or consider them only on abstract level.

Regarding the fact that stationary processes cause $25 \%$ of total $\mathrm{CO}_{2}$-emissions in transportation an approach for detailed consumption analysis on loading unit level is required [13]. Current available simulation solutions are not able to determine GHG-emissions of handling processes. Several simulation approaches considering consumption values for single handling equipment such as floor conveyors. Since these approaches do not include different ambient conditions like topographical characteristics as well as empty or loaded run, this paper considers integration of those aspects to simulation environments for multimodal handling terminals.

\section{Solution Approach}

The presented project is based on a former developed method kit to evaluate GHG-emissions for various transportation modalities. In this project research is focused on the emissions of handling operations in multimodal container terminals. Therefore, five work packages are defined. To meet a high level of accurateness simulation is used to evaluate emission data for different terminal layouts. The estimated values are the system load for the simulation independent $\mathrm{CO}_{2}$-method kit which is developed in Microsoft Excel.

The approach contains a current state analysis. In this step the multimodal container terminals of the industry project partners are analysed concerning layout, modal split and handled loading units. Furthermore, throughput, utilization and seasonal deviations are calculated individually. Depending on the modes of transport the container terminal can be divided into various functional areas according to the connected modalities. This paper focuses on bimodal and trimodal terminal layouts. Figure 2 illustrates all functional areas in a schematic layout.

At second the evaluation of consumption values of various handling equipment of container terminals is concerned. In this case specialist literature is studied and data of manufacturers of handling equipment are collected. Furthermore, all data and information are validated and complemented. 
Therefore, measurements are done which record the consumption for an exemplary early shift. Those measurements take place at the multimodal container terminal facilities of project partners.

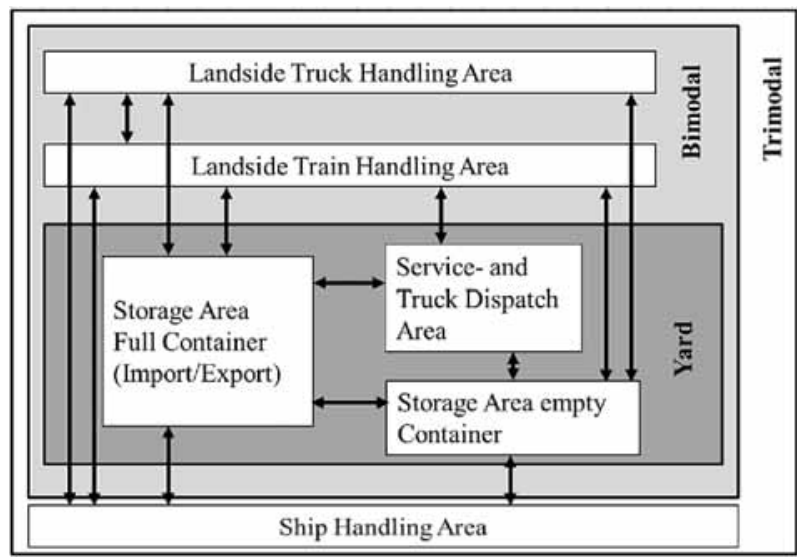

Figure 2: Functional areas of a container terminal.

In the following, consumption affecting factors are determined by deductive analysis. The objective is to higher knowledge about all relevant factors or technical specifications which influence the energy consumption of specific handling equipment. Various discussions during the project about the results lead to the ability to identify emission causing factors of handling operations in terminal layouts. The evaluated results are categorized and furthermore presented to all project partners to evaluate relevant influencing factors.

In recent times the Institute of Transport Logistics developed a tool to run simulations for handling facilities within combined transportation chains. This tool is focussed on simulation and is complemented by a module which allows the evaluation of GHG-emissions. This is an event-driven and module based simulation which maps multimodal container terminals including all stochastic interdependencies of different functional areas in an experimental model. For that purpose, various, appropriate to all evaluated emission causing factors, facility models are analysed by scenario technique. On this way system load for each scenario is generated. Based on those and by help of a key performance indicator catalogue the GHG-emissions are possible to allocate accurately for each loading unit.

The results of all described working steps are gathered in one module for multimodal logistics hubs. This is an advancement of the already existing determination tool for GHG-emissions among the entire transportation chain of containers.
With help of this module it is also possible to evaluate the GHG-emission of a specific terminal layout by filling in all relevant KPI's. The decision to choose Microsoft Excel to develop that method kit is obvious since the first version of this GHG-emission method kit was based on it and Microsoft Excel is the most common program, even in small companies, which don't have to invest a lot to gain benefits from such a method kit.

\section{Integration of Measured Values into the Simulation Environment}

Due to a missing reliable database for energy consumption values of container handling instruments, first of all such a database is meant to be developed. For that purpose, numerous measurements are made at various handling facilities which use different models of gantry and quay cranes of different ages. Those measured values are data load for crane modules in a container terminal simulation. Furthermore, within all measurements a detailed process analysis is realized which is synchronized to the energy consumption analysis to allocate consumption values to single process steps. A whole handling cycle consists of process steps which are shown in Figure 3.

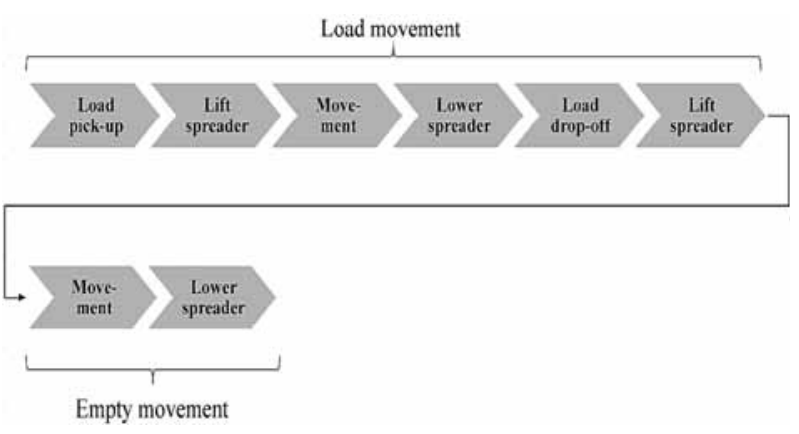

Figure 3: The processes of crane operation.

The illustrated process chain starts with an empty run to reach the next loading unit which requires handling. After positioning above of the loading unit, the spreader is lowered. By closing twist-locks or grippers the crane picks up the loading unit and lifts, to move it to its dedicated position. Once the crane arrived at the loading unit final position it lowers the spreader and reopen spreader or grippers to drop off the loading unit. Finally, the crane lifts the spreader again and is now available for another handling process. 
The measured effective power is allocated to all process steps on a secondly basis and can be integrated in all events in the crane module of the simulation environment. The determination of all GHG- emissions during the transshipment process requires a simulation tool that reflects all subprocesses in an appropriate level of detail.

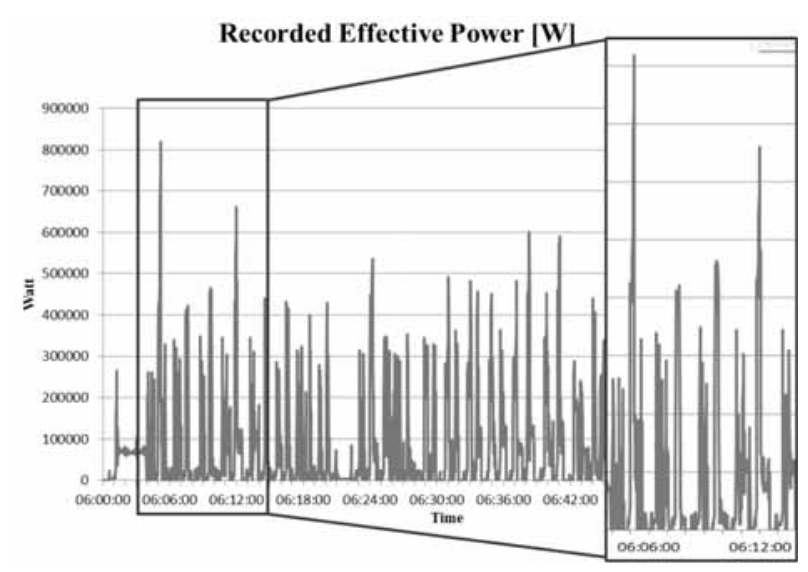

Figure 4: Record of Consumption.

TerminalSim is applicable in that case. It is based on Enterprise Dynamics [12]. TerminalSim is a LowLevel-of-Detail simulation solution for container terminals which contains parameterized modules which are able to map all functional areas of multimodal handling facilities. TerminalSim is integrated to a standard data base connection to provide quick modelling and analysis of different simulation scenario. This allows parameterizing automatically, executing and evaluating each scenario. All crane modules in TerminalSim are expanded by all results of energy consumption measurements [2]. Those values demonstrate differences for energy consumption of cranes regarding their construction year (Figure 4). New and modern cranes with or without energy recovery have lower energy consumption as cranes with an age between one and ten years. Cranes which are older than ten years show the highest values of energy consumption.

To get an idea of the emission causing factors in multimodal container terminals it is essential to seek after all parameters of the cycles in the recorded shifts. Basically, it is the aim to get results for energy consumption data per loading unit. In this context, the parameters of all loading units should be classified to identify emissions of loading units with different technical specifications, weights or handling durations.
Definition of weight classes. Therefore, all loading units are classified to different weight classes to group them and in case to show allocation of emission for each weight class. To be able to analyse all loading units regarding their weights, classes are defined. On that basis all loading units can be assigned to on weight class. Those weight classes are named by the type of loading unit plus the number for the individual weight class.

\begin{tabular}{|c|c|c|c|}
\hline Type & Name & Weight & Name \\
\hline 20 feet & $20^{\circ}$ & empty & $\mathrm{CT}+1$ \\
\hline 40 feet & $40^{\circ}$ & $0<t<=10$ & $\mathrm{CT}+2$ \\
\hline 45 feet & $45^{\circ}$ & $10<t<=20$ & $\mathrm{CT}+3$ \\
\hline High-Cube & $\mathrm{HC}^{*}$ & $20<t$ & $\mathrm{CT}+4$ \\
\hline
\end{tabular}

Figure 5: Container classification.

Figure 5 gives a schematic overview about the definition of classes. Furthermore, those classifications also exist for trailers. In case of trailers, handling in terminals the classification of the loading units regarding their weight is similar and the weight classes remain.

- Example: a 20 feet sized container which carries 21 tons is defined in container class 204

Definition of distance classes. Furthermore, the covered distance for each handled loading unit is focussed to identify possible influence to GHG-emission. Therefore, the covered distances of loaded and empty tours are important for the analysis. Similar to the weight classification also those distances are grouped to distance classes which are able to be examined regarding their GHG-emission.

\begin{tabular}{|c|c|c|c|}
\hline Relation & Distance class & Distance & Class \\
\hline Distance loaded & EL & $<20 \mathrm{~m}$ & $\mathrm{ET}+1$ \\
\hline Distance empty & EI & $20 m<x<60 m$ & $\mathrm{ET}+2$ \\
\hline \multirow[t]{2}{*}{ Distance Cycle } & Eg & $60 m<x<100 m$ & $\mathrm{ET}+3$ \\
\hline & & $>100 \mathrm{~m}$ & $\mathrm{ET}+4$ \\
\hline
\end{tabular}

Figure 6: Distance classification.

In contrast to the weight classes it is beneficial to specify the distances of each handling cycle more detailed. Basically, the total distance of a single handling operation can be separated into a distance covered with load or without load. Within the analysis both components are defined as separate class (Figure 6). Class "EL" represents the covered distance with load and in contrast to that " $E l$ " represents the distance which is need to covered empty. 
This detailed classification is essential, since both distances can differ a lot in one full cycle. It is possible that a resource covers a huge distance loaded and just a very small distance unloaded because the next loading unit is waiting right next to the previous drop-off position. Of course, the situation can occur vice versa. Since the distance is surely an emission causing factor it is part of this analysis to identify if there is a difference in loaded and unloaded movements.

Analysis of energy consumption. Before evaluating the average energy consumption to achieve a statement regarding the GHG-emission of handling operations in multimodal container terminals the key figures which describe the measuring span should be evaluated. Based on those data the average consumption can be identified.

To get this measuring span every terminal is analysed concerning

- Amount of container classes

- Distance classes per loading unit

- Total consumption and consumption per type of loading unit

- Consumption depending on covered distance and weight.

Amount of container classes. For each container terminal where measurements were done statistics state the distribution of the different kinds of unit loads. Those data give a quick overview of the container or trailer mix which is representable for daily operation. In Figure 6, a sample is developed to show how the mix of loading units was mapped.

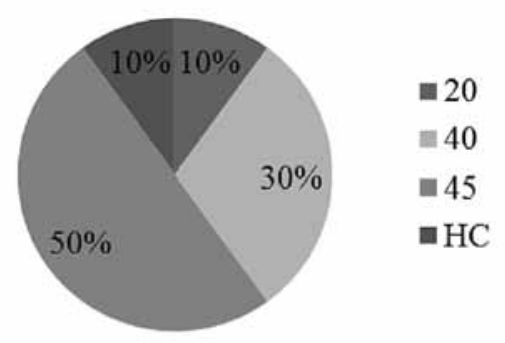

Figure 6: Share of container classes (example).

All those basic parameters about the mix of loading units need to be analyzed in detail. Since a point of concern is the average consumption depending on the weight of a loading unit it is also essential to know the distribution according to the defined weight classes.
Those overviews allow evaluating the representative-ness of the recorded consumption values. By considering the size of all single samples, it is possible to identify outliers or average values. Figure 7 shows a sample distribution in a specified container weight class.

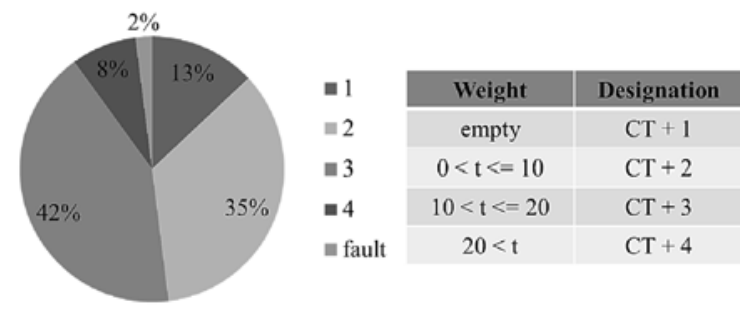

Figure 7: Share of container.

Distance classification. To evaluate more consumption causing factors a point of concern is furthermore the covered distance while handling operations in multimodal container terminals.

After discussions with project partners and operators of container terminals, the distance of the handling operations was identified as possible factor. Taking this into account, an analysis based on the covered distance is meant to turn assumption to statement.

For that reason, each kind of loading unit is also considered according to their individual distance. The distances of the cycles are grouped to specific classes, such as the weight classifications (Figure 8). Furthermore, useful classes were defined to get a detailed insight to the distance depending energy consumption.

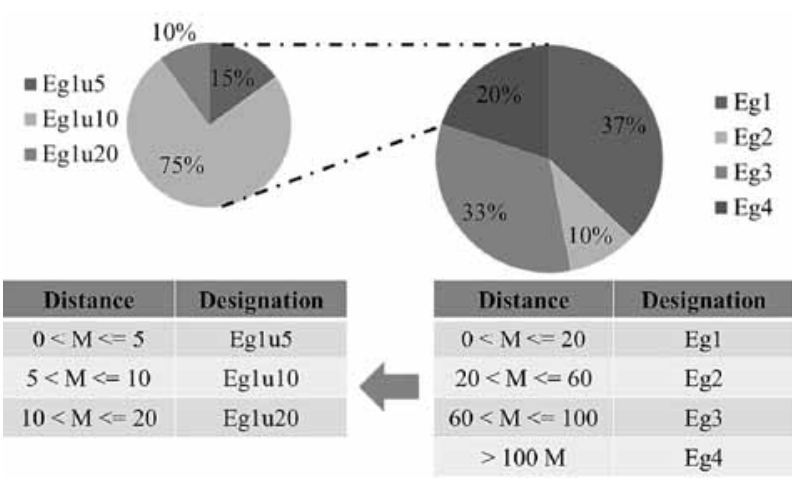

Figure 8: Distance classes. 


\section{Allocation of Energy Consumption with Simulation}

In the course of this paper, total consumption of multimodal handling facilities are aimed to be allocated on loading unit level by considering dependence of different parameters. This is realized using simulation methods.

For this purpose, specific simulation models, based on identified parameters, are developed. Those models are useful to derive consumption values for different handling situations. This is essential, since process analysis is done only for exemplary early shifts and those values do not represent general conclusions regarding handling volume and loading unit structure.

To ensure provision of long term oriented (> 1 year) consumption values, simulation is needed to display consumption by simultaneous allocation on loading unit level.

Content of this article are results of the first analyzed terminal. The considered terminal is equipped with a gantry crane and various reach stackers for trimodal handling operations. The handling area got a length of $420 \mathrm{~m}$ and is $135 \mathrm{~m}$ wide. This area is completely covered by a gantry crane. Furthermore, the area consists of a quay side handling area for container vessels, a container depot, 4 tracks for trains which cover $350 \mathrm{~m}$ length and loading areas for trucks. All handlings from landside to seaside area and vice versa are done by the gantry crane. In opposition loading and unloading of trains hat the railroad tracks are advised to the reach stackers. The crane only supports the reach stacker units only in case of free capacities or meeting acceptance.

The terminal handles TEU 150.000 per annum. Here, the contribution focusses the handlings proceeded by the gantry crane. Reach stackers are included in this project at later stage. Duration handling operations of the gantry crane, backing-in times of trains and trucks are captured as well as provisioning time of trains and the time of arrival and departure of every ship during one week of employees of the terminal. Based on these measurements, stochastic distribution functions were defined and implemented to the simulation model as element for time consumption.

Based on historical values a data set of 2014 is considered to include seasonal effects in simulation. In the next phase 50 simulation runs are executed. By discussion with experts, at the handling facilities location, the results were validated.
Simulations as well as measurements state no influence to energy consumption by the weight of loading units in handling operations. A remarkable influencing factor is the handling distance of loading units. The result of analysis shows a high influence of moving distance to energy consumption of gantry cranes. To get those results more detailed, distance classes are defined according to an ABC-analysis approach. Figure 9 show results of different distance classes. Consumption is increasing according to distance.
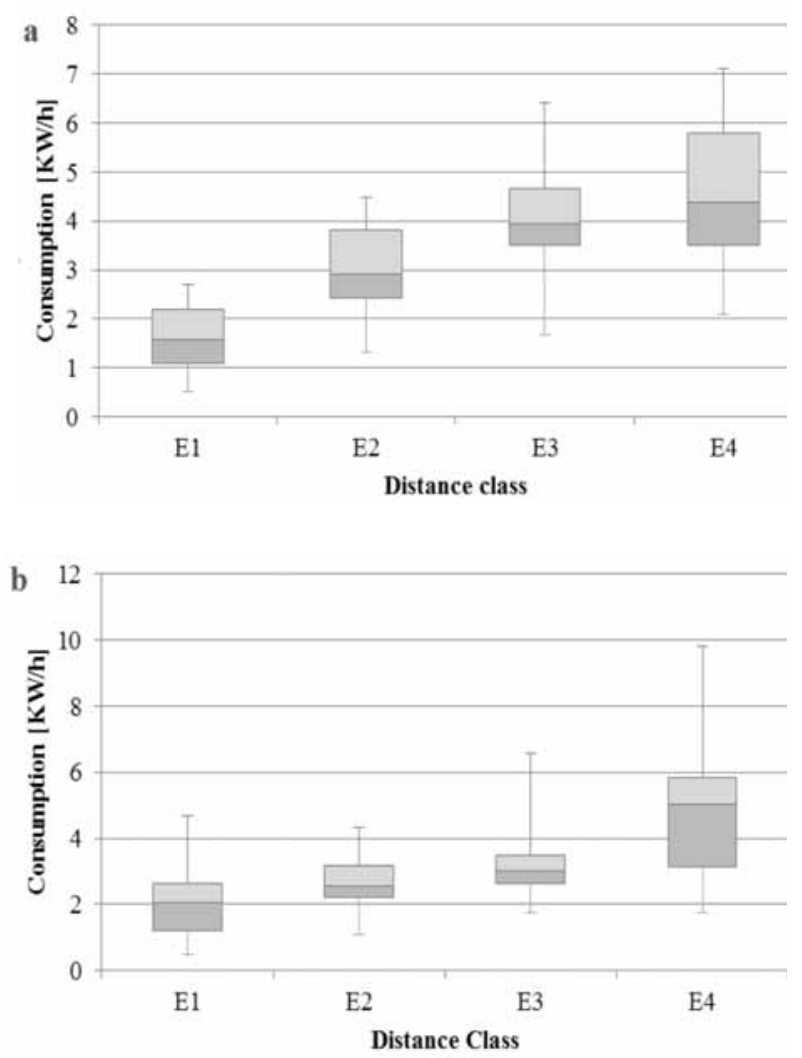

Figure 9: Consumption per Distance class for 20-FeetContainer (a) and 40-Feet-Container (b).

- Class E1 - up to 20m

- Class E2 - $21 \mathrm{~m}$ to $60 \mathrm{~m}$

- Class E3 - 61m to $100 \mathrm{~m}$

- Class E4 - more than $100 \mathrm{~m}$

Based on the results of simulation and furthermore by including environmental influences and especially relocations, following consumption values were identified (Table 1). 


\begin{tabular}{|c|c|c|c|c|c|}
\hline Container & $\varnothing$-time & $\begin{array}{l}\emptyset- \\
\text { KWh }\end{array}$ & $\begin{array}{l}\varnothing- \\
€ / K W h\end{array}$ & $\begin{array}{l}\varnothing-\mathrm{KG} \\
\mathrm{CO}_{2} / \mathrm{KWh}\end{array}$ & $\begin{array}{l}\varnothing- \\
\text { distance }[\mathrm{m}]\end{array}$ \\
\hline $\begin{array}{l}20^{\prime \prime} \\
\text { empty }\end{array}$ & $0: 02: 58$ & 3.65 & 0.40 & 1.56 & 88.84 \\
\hline $\begin{array}{l}40^{\prime \prime} \\
\text { empty }\end{array}$ & $0: 02: 47$ & 3.09 & 0.34 & 1.32 & 66.68 \\
\hline $\begin{array}{l}20 " \\
\text { light }\end{array}$ & 0:03:00 & 4.02 & 0.44 & 1.72 & 124.14 \\
\hline $\begin{array}{l}40 " \\
\text { light }\end{array}$ & 0:03:30 & 3.48 & 0.38 & 1.49 & 84.60 \\
\hline $\begin{array}{l}20 " 1 \\
\text { medium }\end{array}$ & $0: 02: 50$ & 3.14 & 0.35 & 1.34 & 180.35 \\
\hline $\begin{array}{l}40^{\prime \prime} \\
\text { medium }\end{array}$ & 0:05:51 & 3.63 & 0.40 & 1.55 & 72.06 \\
\hline 20" heavy & 0:03:00 & 4.19 & 0.46 & 1.79 & 95.23 \\
\hline 40" heavy & $0: 02: 42$ & 3.82 & 0.42 & 1.63 & 123.99 \\
\hline
\end{tabular}

Table 1: Allocated energy consumption on container-level.

On average a single container handling cost $€ 0.40$ and emits $1.55 \mathrm{~kg} \mathrm{CO}_{2}$ which corresponds to the ecological balance sheet. This proofs the simulation approach as valid. By use of this presented method it is now possible to allocate more precisely emission values for single types of loading units. This provides numerous advantages for terminal operators in case of marketing, optimization or differentiated accounting activities.

\section{Conclusion}

Within this research project power consumption and influencing factors on multimodal transhipment terminals have been identified. The results showed that handling distances are the most influencing factors regarding energy consumption. Hence, to save energy and costs it is necessary to reduce handling distances. Secondly, the results showed that relocations are an important factor that should be reduced. Each relocation action is causing additional energy consumption.

In further steps, the results will be expanded to cover additional resource consumption values and terminal layouts. Furthermore, nonspecific terminal resources e.g. lighting systems will be added.

The results are being integrated in the Microsoft Excel based $\mathrm{CO}_{2}$-allocation tool and extend the existing $\mathrm{CO}_{2}$-method kit to empower sme logistic service provider to determine GHG-emissions of handling operations in multimodal container terminals.
Acknowledgment. This article presents results of the research project " $\mathrm{CO}_{2}$-allocation - multimodal logistic hubs: Enhancement of the $\mathrm{CO}_{2}$-method kit for an exact determination of environmental effects in multimodal logistic hubs". The research project is encouraged by the Federal Ministry of Economic Affairs and Energy through AiF and on behalf of BVL (Bundesvereinigung Logistik) - grant agreement 17961 N/1.

\section{References}

[1] Bundesverband Güterkraftverkehr Logistik und Entsorgung (BGL) e.V. Modal Split im Güterverkehr 19502014 nach Tonnenkilometern. Available: http://www.bgl-ev.de/images/daten/verkehr/ modalsplittkm_tabelle.pdf, updated: January 2016.

[2] Clausen, U. Maßnahmen im mobilen Bereich - Ergebnisse von StaMoLo und deren Umsetzung in der Praxis. In: Klimaschutz in der Logistik: Treibhausgase mindern - Kosten senken. Bonn. 2008.

[3] Clausen, U, Kaffka, J. Modeling of Handling Task Sequencing to Improve Crane Control Strategies in Container Terminals. In: Proceedings of the 2012 Winter Simulation Conference. 2012: pp. 4673-4781.

[4] Clausen, U, Kaffka, J, Meier, F. CONTSIM - Container Terminal Management with Simulation. In: Proceedings of EWGT2012 - 15th Meeting of the EURO Working Group on Transportation. 2012; pp. 332-340.

[5] Deutsches Institut für Normung e.V. DIN EN ISO 14040 (2006-10-00): Environmental management -Life cycle assessment -Principles and framework (ISO 14040:2006); German and English version EN ISO 14040:2006. Berlin: Beuth, 00.10.2006.

[6] Deutsches Institut für Normung e.V. DIN EN ISO 14044 (2006-10-00): Environmental management - Life cycle assessment - Requirements and guidelines (ISO 14044:2006); German and English version EN ISO 14044:2006. Berlin: Beuth, 00.10.2006.

[7] Deutsches Institut für Normung e.V. DIN EN (ISO/DIS 16258: 2012). Methodology for calculation and declaration of energy consumption and GHG emissionsof transport services (freight and passengers). Berlin: Beuth, 00.03.2013.

[8] Duinkerken, MB, Dekker, R, Kurstjens, ST, Ottjes, J A, Dellaert, NP. Comparing transportation systems for inter-terminal transport at the Maasvlakte container terminals. In: Günther, HO, Kim, KH (Ed.): Container Terminals and Cargo System. Springer, Berlin, 2007, pp. 37-61.

[9] European Comission. (2015). Reducing emissions from transport [Online]. Available: http://ec.europa.eu/clima/policies/transport/index_en.htm 
[10] Kaffka, J, Mest, L. Optimierung von Binnenhafencontainerterminals mittels Simulation. In: Schifffahrt und Technik. SUT, Sankt Augustin, 2010, p. 85.

[11] Kaffka, J, Clausen, U, Chmielewski, A, Schütte, T. $O p$ timization and simulation of operating strategies for container terminals. In: General Proceedings of the 12th WCTR. 2010.

[12] Kaffka, J, Clausen, U, Stein, S. Revealing Gaps in the Material Flow of Inland Port Container Terminals alongside the Danube with Simulation. In: Proceedings of the 2014 Winter Simulation Conference, 2014, pp. 1807-1818.

[13] Kranke, A, Schmied, M, Schön, A.D. CO2-Berechnung in der Logistik. Datenquellen, Formeln, Standards. 1st Edition. Munich: Vogel; 2011.

[14] Lampe, H. Untersuchung von Dispositionsentscheidungen in Umschlagterminals des Kombinierten Verkehrs Schiene/Straße. Dortmund: Praxiswissen; 2006.

[15] Lee, BK., Jung, BJ, Kim, KH, Park, SO, Seo, JH. A Simulation Study for Designing a Rail Terminal in a Container Port. In: Proceedings of the 2006 Winter Simulation Conference. 2006; pp. 1388-1397.

[16] McKinnon, A, Piecyk, M. Measuring and Managing $\mathrm{CO}_{2}$-Emissions of European Chemical Transport. Edited by Cefic -The European Chemical Industry Council. Heriot-Watt University, Logistics Research Centre. Edinburgh. Available online at www.cefic.org. 2010.
[17] OECD. Reducing Transport Greenhouse Gas Emissions [Online]. 2012. Available: www.itf-oecd.org/sites/ default/files/docs/10ghgtrends.pdf.

[18] Rabe, M, Deininger, M. State of Art and Research Demands for Simulation Modeling of Green Supply Chains. In: International Journal of Automation Technology. 2012, 6(3): Fuji Technology Press, Tokyo, pp. 296-303.

[19] Rabe, M, Gutenschwager, K, Fechteler, T, Sari, U. A Data Model for Carbon Footprint Simulation in Consumer Goods Supply Chains. In: Proceedings of the 2013 Winter Simulation Conference. 2013; pp. 2677-2688.

[20] Schmied, M, Knörr, W. Berechnung von Treibhausgasemissionen in Spedition und Logistik. Begriffe, Methoden, Beispiele. Edited by Deutscher Speditions- und Logistikverband e.V. Öko-Institut e.V.; ifeu - Institut für Energie- und Umweltforschung Heidelberg GmbH. Cologn, 2011.

[21] Thomas, J. Umweltökonomische Analyse des Güterverkehrs 1995 bis 2010. In: Wirtschaft und Statistik. 2012, 5: Statistisches Bundesamt, Wiesbaden, pp. 503-514.

[22] Umweltbundesamt (UBA). Treibhausgasausstoß in Deutschland 2012 - vorläufige Zahlen aufgrund erster Berechnungen und Schätzungen des Umweltbundesamtes. February 2013. 
